\title{
Anisotropic plasmons in a two-dimensional electron gas with spin-orbit interaction
}

\author{
S. M. Badalyan,,$^{1,2, *}$ A. Matos-Abiague, ${ }^{2}$ G. Vignale, ${ }^{3}$ and J. Fabian ${ }^{2}$ \\ ${ }^{1}$ Department of Radiophysics, Yerevan State University, 1 A. Manoukian Street, Yerevan 375025, Armenia \\ ${ }^{2}$ Department of Physics, University of Regensburg, 93040 Regensburg, Germany \\ ${ }^{3}$ Department of Physics and Astronomy, University of Missouri-Columbia, Columbia, Missouri 65211, USA
}

(Received 9 February 2009; revised manuscript received 15 April 2009; published 7 May 2009)

\begin{abstract}
Spin-orbit coupling-induced anisotropies of plasmon dynamics are investigated in two-dimensional semiconductor structures. The interplay of the linear Bychkov-Rashba and Dresselhaus spin-orbit interactions drastically affects the plasmon spectrum: the dynamical structure factor exhibits variations over several decades, prohibiting plasmon propagation in specific directions. While this plasmon filtering makes the presence of spin-orbit coupling in plasmon dynamics observable, it also offers a control tool for plasmonic devices. Remarkably, if the strengths of the two interactions are equal, not only the anisotropy but all the traces of the linear spin-orbit coupling in the collective response disappear.
\end{abstract}

DOI: 10.1103/PhysRevB.79.205305

PACS number(s): 71.45.Gm, 72.25.Dc, 73.21.Fg, 73.63.Hs

Spin-orbit coupling in semiconductor heterostructures has received wide attention recently-it has been investigated as a source of new fundamental spin physics as well as a control interaction in spintronics applications. ${ }^{1,2}$ Two spin-orbit terms are relevant in zinc-blende systems exemplified by two-dimensional GaAs or InAs electron gases: the Bychkov-Rashba ${ }^{3}$ interaction (coupling constant $\alpha$ ) which is due to the structure inversion asymmetry and the Dresselhaus interaction ${ }^{4,5}$ (coupling constant $\beta$ ) which is due to the bulk inversion asymmetry. ${ }^{2}$ Alone these interactions lead to an isotropic single particle and plasmon spectrum. Taken together, they imprint the underlying heterostructure anisotropy onto the single- and many-particle properties. Most studies of the spin-orbit coupling effects have been on the single-particle level. While the presence of spin-orbit coupling leads to such notorious effects as spin relaxation ${ }^{2}$ or spin Hall currents, ${ }^{6,7}$ fascinating phenomena originate from the interplay of the Bychkov-Rashba and Dresselhaus terms. The interplay often leads to pronounced anisotropies, ${ }^{8-14}$ but this is not a rule. ${ }^{15}$

Recently several many-body effects important for spin properties of semiconductor nanosystems have been studied in two-dimensional electron system (2DES). ${ }^{16-18}$ One of the key phenomena due to spin-orbit interaction (SOI) in manyspin systems is the generation of the interchirality subband electron-hole continuum. However, the dispersive and dissipative modifications induced by individual (Bychkov-Rashba or Dresselhaus) SOI are difficult to observe in experimenttheir effect is isotropic and proportional to the small SOI coupling. ${ }^{19-26}$ In real samples the interplay of different SOI mechanisms takes place and as we show here, it results in the striking anisotropy effect on the spectral properties of collective excitations in 2DES. This qualitatively strong effect can serve as a valuable tool to facilitate the observation and exploitation of usually weak SOI effects on many-body properties of 2DES.

An important outcome of our theory is the prediction of plasmon directional filtering: the interplay of the spin-orbit couplings leads to plasmon overdamping (blocking) in certain special directions of propagation and for certain magnitudes of the wave vector. This may be surprising at first sight, given that the spin-orbit effects on the plasmon disper- sion and on the electron-hole excitation energies are in themselves quite small. However even small energy shifts are sufficient, at these special wave vectors, to move the plasmon in or out of resonance with electron-hole excitations, thus producing a large effect on the plasmon damping. By scanning for plasmons in different directions, this distinct absence of propagation in certain directions should be experimentally verifiable since the dynamical structure factor varies by orders of magnitude as a function of the propagation angle. In addition to making the spin-orbit presence experimentally visible, the anisotropy is attractive for plasmonics designs as a substitute for surface patterning to achieve directional plasmon propagation. ${ }^{27}$ This prospect is enforced by the possibility to control_-even turn on and off_-plasmon propagation: both $\alpha$ and $\beta$ can be tuned by external gates ${ }^{1}$ (see also Refs. 28 and 29) allowing for the anisotropy to be tailored. In fact, the anisotropy vanishes (filtering turned off) for $\alpha= \pm \beta$. More surprising, in this case the (linear) spinorbit couplings play no role in plasmon dynamics - the isotropic contributions by the individual spin-orbit terms cancel each other.

We calculate the effect of joint Bychkov-Rashba and Dresselhaus SOI on the propagation of plasmons in the (001) plane of a zinc-blende semiconductor heterostructure. We consider samples at low temperatures with high-density 2DES where the kinetic energy of electrons dominates the Coulomb potential energy. In this regime it is legitimate to neglect the effect of exchange and correlations in treating plasmon excitations. We use the random-phase approximation (RPA) (Ref. 30) and calculate the anisotropic Lindhard polarization function for a given wave vector $\mathbf{q}$ and frequency $\omega$. The space in which the imaginary part of the Lindhard function differs from zero is known as the electronhole continuum (EHC) (Ref. 30) for it describes the spectrum of electron-hole excitations. The interplay of the Bychkov-Rashba and Dresselhaus SOI leads to the appearance of several subregions of the EHC separated by boundaries across which the imaginary part of the dielectric function exhibits sharp variations. An interesting effect arises when the frequency of a plasmon of a given $q$ but variable propagation direction crosses these boundaries: the sudden rise in the density of electron-hole excitations causes strong 

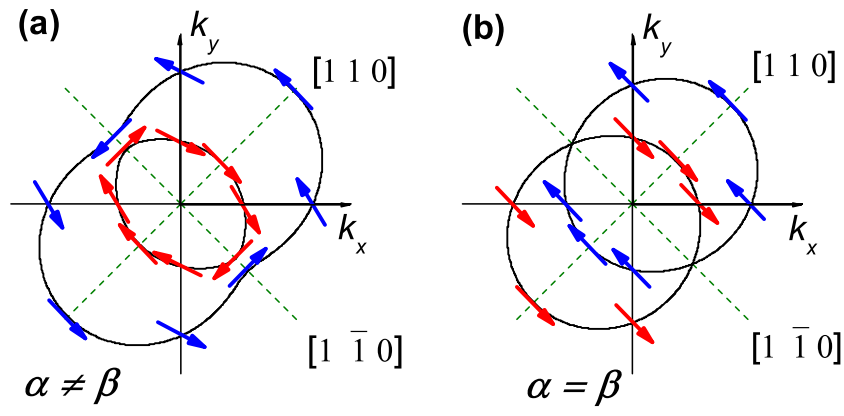

FIG. 1. (Color online) Fermi contours in the momentum plane $\left(k_{x}, k_{y}\right)$ for a spin-orbit interaction of the form given in Eq. (1): (a) $\alpha \neq \beta$ and (b) $\alpha=\beta$. The arrows indicate the spin direction.

Landau damping, actually overdamping the plasmons over a range of wave-vector orientations. This anisotropy of the plasmon spectrum should be observable through the pronounced anisotropy of the dynamical structure factor, as shown below.

Our spin-orbit interaction Hamiltonian is ${ }^{2}$

$$
H_{\mathrm{SOI}}=\alpha\left(\hat{\sigma}_{x} k_{y}-\hat{\sigma}_{y} k_{x}\right)+\beta\left(\hat{\sigma}_{x} k_{x}-\hat{\sigma}_{y} k_{y}\right),
$$

where $\hat{\sigma}_{x, y}$ are the Pauli matrices, $\vec{k}$ is the inplane electron momentum with magnitude $k$, and polar angle $\phi_{\mathbf{k}}$. The eigenvectors of the Hamiltonian $H=H_{0}+H_{\text {SOI }}$ with $H_{0}=k^{2} / 2 m^{*}$ $\left(m^{*}\right.$ is the electron effective mass and $\hbar=1$ ) are

$$
\Psi_{\mu}(\vec{r})=\frac{1}{\sqrt{2}}\left(\begin{array}{c}
i e^{-i \varphi} \\
\mu
\end{array}\right) \frac{e^{i \vec{k} \vec{r}}}{\sqrt{A}},
$$

corresponding to the single-particle spin-split branches of the electron energy

$$
E_{\mu}(\vec{k})=\frac{1}{2 m^{*}}\left\{\left[k+\mu \xi\left(\rho, \theta, \phi_{\mathbf{k}}\right)\right]^{2}-\xi\left(\rho, \theta, \phi_{\mathbf{k}}\right)^{2}\right\}
$$

labeled by the chirality $\mu= \pm 1 ; A$ is the area of $2 \mathrm{DES}$. The phase of the spinor in Eq. (2) is $\varphi\left(\alpha, \beta, \phi_{\mathbf{k}}\right)=\operatorname{Arg}\left[\alpha e^{i \phi_{\mathbf{k}}}\right.$ $\left.+i \beta e^{-i \phi_{\mathbf{k}}}\right]$ and the angle dependent Rashba-Dresselhaus momentum is

$$
\xi\left(\rho, \theta, \phi_{\mathbf{k}}\right)=\rho \sqrt{1+\sin (2 \theta) \sin \left(2 \phi_{\mathbf{k}}\right)}
$$

with amplitude $\rho=m^{*} \sqrt{\alpha^{2}+\beta^{2}}$. The angle parameter $\theta$, defined as $\tan \theta=\beta / \alpha$, describes the relative strength of the Bychkov-Rashba and Dresselhaus SOI. The Fermi momenta of the subbands Eq. (3) are also angle dependent,

$$
k_{F}^{\mu}\left(\rho, \theta, \phi_{\mathbf{k}}\right)=\sqrt{2 m E_{F}+\xi\left(\rho, \theta, \phi_{\mathbf{k}}\right)^{2}}-\mu \xi\left(\rho, \theta, \phi_{\mathbf{k}}\right),
$$

where the total carrier density $n$ determines the Fermi energy, $E_{F}=\left(\pi n-\rho^{2}\right) / m^{*}$. Figure 1 illustrates the energy spectrum of the chirality subbands and the anisotropy of the Fermi contour (note that the Fermi energy can be negative).

The Lindhard polarization function ${ }^{30}$ in the presence of SOI is defined as a sum over chirality indices $\Pi(\vec{q}, \omega)$ $=\sum_{\mu, \nu= \pm 1} \Pi_{\mu \nu}(\vec{q}, \omega)$ with

$$
\begin{aligned}
\prod_{\mu \nu}(\vec{q}, \omega)= & \int \frac{d \vec{k}}{(2 \pi)^{2}} \frac{f\left[E_{\mu}(\vec{k})\right]-f\left[E_{\nu}(\vec{k}+\vec{q})\right]}{E_{\mu}(\vec{k})-E_{\nu}(\vec{k}+\vec{q})+\omega+i 0} \\
& \times \mathcal{F}_{\mu \nu}(\vec{k}, \vec{k}+\vec{q}),
\end{aligned}
$$

where $f\left[E_{\mu}(\vec{k})\right]$ is the Fermi distribution function. The form factors $\mathcal{F}_{\mu \nu}(\vec{k}, \vec{k}+\vec{q})$ are given by

$$
\mathcal{F}_{\mu \nu}(\vec{k}, \vec{k}+\vec{q})=\frac{1}{2}\left[1+\mu \nu \cos \left(\Delta \varphi_{\mathbf{q}}\right)\right],
$$

where we define $\Delta \varphi_{\mathbf{q}}=\varphi\left(\alpha, \beta, \phi_{\mathbf{k}}\right)-\varphi\left(\alpha, \beta, \phi_{\mathbf{k}+\mathbf{q}}\right)$. Notice that in contrast to the case of pure Bychkov-Rashba or pure Dresselhaus SOI, here the polarization function depends both on the magnitude, $q$, and orientation, $\phi_{\mathbf{q}}$, of the wave vector $\vec{q}$. Making the replacement $\vec{k} \rightarrow-\vec{k}-\vec{q}$ in the term of Eq. (7) with $f\left[E_{\nu}(\vec{k}+\vec{q})\right]$ and regrouping, we can represent the polarization function in the compact form $\Pi(\vec{q}, \omega)$ $=\Sigma_{\mu, \nu, \lambda= \pm 1} \Pi_{\mu, \nu}^{\lambda}(\vec{q}, \omega)$, where

$$
\prod_{\mu, \nu}^{\lambda}(\vec{q}, \omega)=\int \frac{d \vec{k}}{(2 \pi)^{2}} \frac{f\left[E_{\mu}(\vec{k})\right] \mathcal{F}_{\mu \nu}(\vec{k}, \vec{k}+\vec{q})}{E_{\mu}(\vec{k})-E_{\nu}(\vec{k}+\vec{q})+\lambda(\omega+i 0)} .
$$

Exploiting further the symmetry of the problem in the limit of zero temperature we reduce the polarization function to the following expression

$$
\prod(\vec{q}, \omega)=\frac{g}{4 \pi} \sum_{\mu, \lambda} \int_{0}^{2 \pi} d \phi_{\mathbf{k}} \int_{0}^{v_{F, \mu}} d v \frac{v\left(e_{\mu, \lambda}-d_{\mu} v\right)}{a_{\mu}\left(v-v_{\mu, \lambda}^{+}\right)\left(v-v_{\mu, \lambda}^{-}\right)} .
$$

Here we have defined the dimensionless Fermi wave vector $v_{F, \mu}=\sqrt{1-r^{2}+\bar{\xi}_{\mathbf{k}}^{2}}-\mu \bar{\xi}_{\mathbf{k}}$ and the functions $v_{\mu, \lambda}^{ \pm}=\left(-b_{\mu, \lambda}\right.$ $\left.\pm \sqrt{b_{\mu, \lambda}^{2}-4 a_{\mu} c_{\lambda}}\right) / 2 a_{\mu}$ with

$$
\begin{gathered}
a_{\mu} \equiv x \cos \left(\phi_{\mathbf{k}}-\phi_{\mathbf{q}}\right)\left[x \cos \left(\phi_{\mathbf{k}}-\phi_{\mathbf{q}}\right)-\mu \bar{\xi}_{\mathbf{k}}\right] \\
b_{\mu, \lambda} \equiv-x\left\{\left[r^{2}+2\left(\lambda y-x^{2}\right)\right] \cos \left(\phi_{\mathbf{k}}-\phi_{\mathbf{q}}\right)\right. \\
\left.+r^{2} \sin (2 \theta) \sin \left(\phi_{\mathbf{k}}+\phi_{\mathbf{q}}\right)\right\}+\mu\left(\lambda y-x^{2}\right) \bar{\xi}_{\mathbf{k}}, \\
c_{\lambda} \equiv\left(\lambda y-x^{2}\right)^{2}-x^{2} \bar{\xi}_{\mathbf{q}}^{2} \\
d_{\mu} \equiv x \cos \left(\phi_{\mathbf{k}}-\phi_{\mathbf{q}}\right)-\mu \bar{\xi}_{\mathbf{k}},
\end{gathered}
$$

$e_{\mu, \lambda} \equiv \lambda y-x^{2}+\frac{\mu r^{2} x}{\bar{\xi}_{\mathbf{k}}}\left[\cos \left(\phi_{\mathbf{k}}-\phi_{\mathbf{q}}\right)+\sin \left(\phi_{\mathbf{k}}+\phi_{\mathbf{q}}\right) \sin (2 \theta)\right]$

Here $g=m^{*} / 2 \pi$ is the density of states at the Fermi level and we have introduced the dimensionless quantities $x=q / 2 k_{F}$, $y=(\omega+i 0) / 4 \varepsilon_{F}, \quad v=k / k_{F}, \quad r=\rho / k_{F}$, and $\bar{\xi}_{\mathbf{k}}=\xi\left(\rho, \theta, \phi_{\mathbf{k}}\right) / k_{F}$ with $\varepsilon_{F}=k_{F}^{2} / 2 m^{*}$ and $k_{F}=\sqrt{2 m^{*} E_{F}+\rho^{2}}$. The integration over $v$ can be done analytically, yielding 

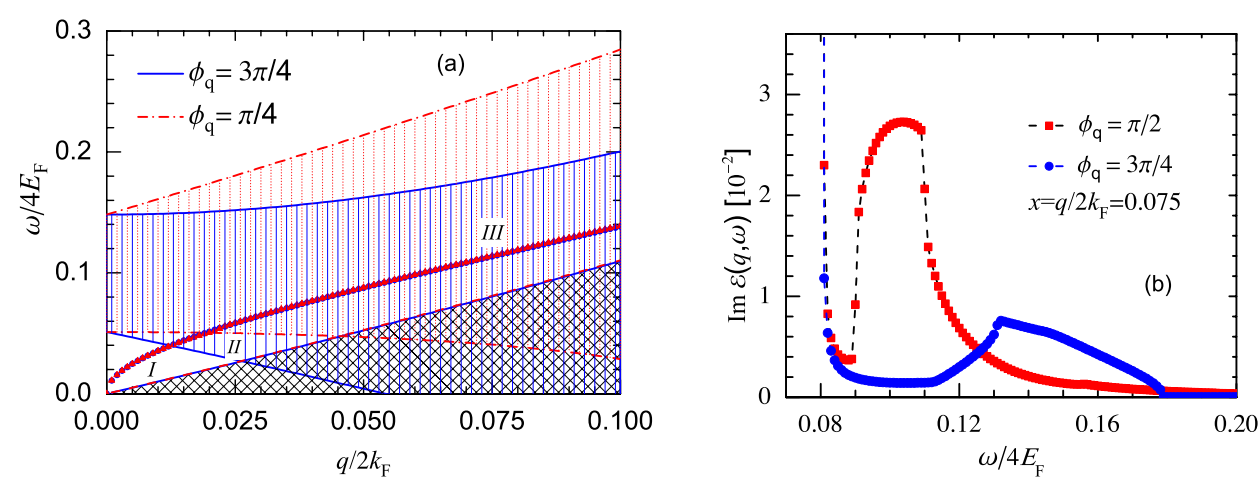

FIG. 2. (Color online) (a) The intra- and interchirality EHC in the $\omega-q$ plane for two different momentum orientations, $\phi_{\mathbf{q}}=\pi / 4$ and $3 \pi / 4$. The symbols show the plasmon dispersions (see text). (b) The imaginary part of the dielectric function vs energy for the fixed momentum magnitude $q$ $=0.15 k_{F}$ and for $\phi_{\mathbf{q}}=\pi / 2$ and $3 \pi / 4$, shown as square and round symbols, respectively.

$$
\begin{aligned}
\prod(\vec{q}, \omega)= & -\frac{g}{4 \pi} \sum_{\mu, \lambda} \int_{0}^{2 \pi} d \phi_{\mathbf{k}}\left\{\frac{d v_{F}}{a}+\frac{1}{a\left(v^{+}-v^{-}\right)}\right. \\
& \times\left[v^{+}\left(e-d v^{+}\right) \ln \frac{v^{+}}{v^{+}-v_{F}}\right. \\
& \left.\left.-v^{-}\left(e-d v^{-}\right) \ln \frac{v^{-}}{v^{-}-v_{F}}\right]\right\} .
\end{aligned}
$$

The integration over $\phi_{\mathbf{k}}$ is performed numerically. The derived formula (15) is exact. In the limit of $\theta=0$ (pure Bychkov-Rashba SOI) we recover the previous results by Pletyukhov and Gritsev ${ }^{19}$ and in the limit of $r=0$ (no SOI) we recover the classic result by Stern. ${ }^{31}$

For the actual calculations we use materials parameters suitable for InAs quantum wells with realistic values of the SOI parameters, $r=0.1$ and $\theta=\pi / 8$ corresponding to the ratio of SOI strengths $\alpha / \beta \approx 2.4$ from Ref. 29. We take the electron density $n=2.55 \times 10^{11} \mathrm{~cm}^{-2}\left(E_{F} \approx 302 \mathrm{~K}\right)$ and the effective transverse width of the quantum well $d=15 \mathrm{~nm}$.

Figure 2(a) shows the EHC regions and the plasmon dispersions for different values of the angle $\phi_{\mathbf{q}}$. The anisotropy of the intrachirality EHC (the dense-hatched region) and of the plasmon dispersions is a small effect and hardly seen on the scale of figure. Meantime, the interchirality EHC is strongly anisotropic (in the long-wavelength limit the anisotropy vanishes). Figure 2(b) shows the imaginary part of the dielectric function vs energy for the fixed momentum magnitude and different orientations. As seen, not only the boundaries of EHC but also the dissipation properties within EHC are strongly anisotropic. In the region near the plasmon energy, $\omega / 4 E_{F} \approx 0.1$ for $q=0.15 k_{F}$, the imaginary part for $\phi_{\mathbf{q}}=3 \pi / 4$ is strongly suppressed with respect to its value for $\phi_{\mathbf{q}}=\pi / 2$.

To calculate the plasmon dispersion we solve for zeros of the real part of the RPA dielectric function

$$
\varepsilon(\vec{q}, \omega)=1-v(q) \prod(\vec{q}, \omega),
$$

where $v(q)=2 \pi e^{2} /\left(\kappa_{0} q\right) F(q d)$ is the bare Coulomb interaction with $\kappa_{0}=14.55$, the static dielectric constant of InAs. For the form factor $F(q d)$ we use the formula

$$
F(\eta)=\frac{8 \pi^{2}+3 \eta^{2}}{\eta\left(4 \pi^{2}+\eta^{2}\right)}-\frac{32 \pi^{4}\left(1-e^{-\eta}\right)}{\eta^{2}\left(4 \pi^{2}+\eta^{2}\right)^{2}}
$$

which takes into account the transverse width $d$ of the quantum well but its asymmetric shape. The form factor has a strong effect on the Coulomb interaction strength ${ }^{17}$ - it goes as $1-\left(1 / 3-5 / 4 \pi^{2}\right) q d$ in the long-wavelength limit $q d \rightarrow 0$ and as $3 /\left(4 \pi^{2} q d\right)$ in the opposite limit $q d \rightarrow \infty$.

There are three distinct regions of EHC and the plasmon dispersions, as seen in Fig. 2(a). In region I, which corresponds to small $q$, the areas of inter- and intrachirality subband transitions are well separated. The plasmon energy is located within the gap between these EHC regions: these plasmons are not damped. The plasmons here exhibit only a SOI induced dispersion as a function of its propagation orientation. At such small $q$, however, the anisotropy is not significant and eventually vanishes in the long-wavelength limit.

At larger values of $q$, in the region III in Fig. 2(a), the plasmon dispersion enters EHC, triggering the phenomenon known as Landau damping, i.e., decay into electron-hole pairs. In this regime the EHC is made up of several overlapping subregions (associated with the discrete quantum indices $\mu$ and $\lambda$ ) separated by sharp boundaries. The imaginary part of the dielectric function (proportional to the spectral density of electron-hole pairs) exhibits sharp variations across these boundaries, resulting in unexpectedly strong angular dependence of plasmon damping. In Fig. 3 we follow the evolution of the plasmon frequency as a function of the angle $\phi_{q}$ from 0 to $\pi$ for $q=0.15 k_{F}$. A sharp boundary be-

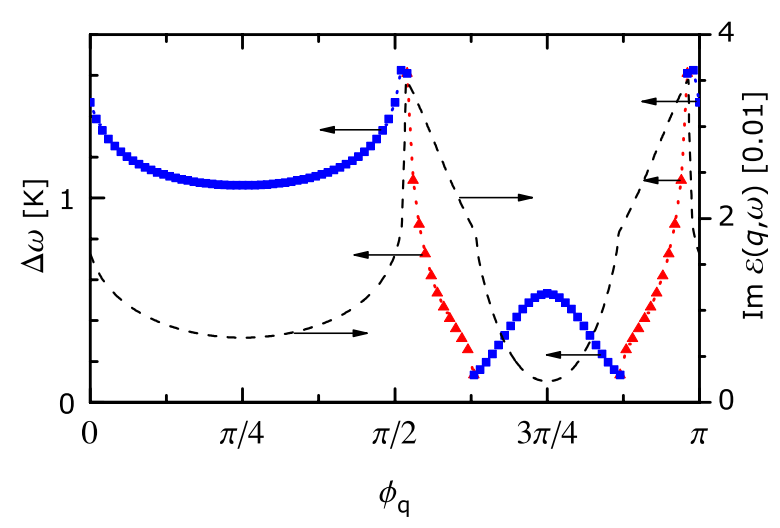

FIG. 3. (Color online) The SOI induced energy dispersion of the plasmon vs its propagation direction for $q=0.15 k_{F}$ (the left axis and square symbols). The parts of the spectrum which do not represent plasmon excitations (see text) are shown as triangle symbols. The dashed line plots the imaginary part of the dielectric function (the right axis) for $q=0.15 k_{F}$ and $\Delta \omega \equiv \omega-\omega_{0}=1.2 \mathrm{~K}$ where $\omega_{0}$ $\approx 0.45 E_{F}$. 


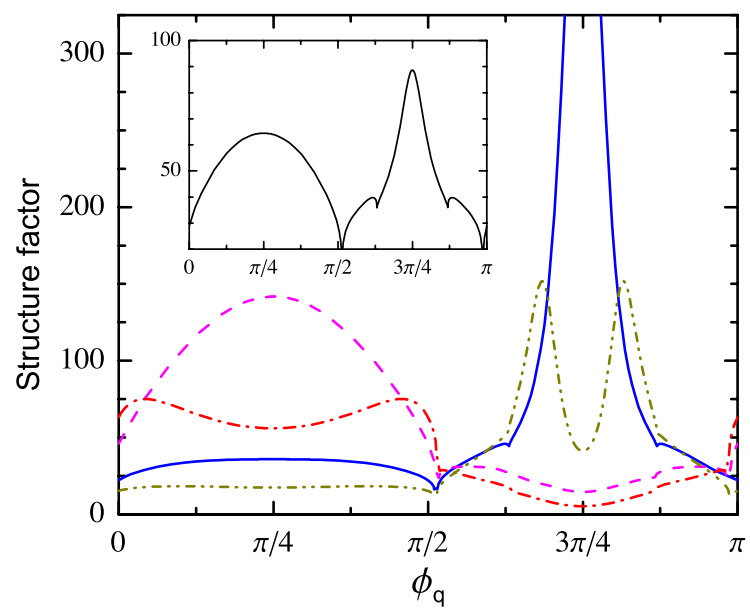

FIG. 4. (Color online) The dynamical structure factor vs $\phi_{\mathbf{q}}$ for $q=0.15 k_{F}$. The solid and dashed lines correspond, respectively, to the local maximum at $\Delta \omega \approx 0.5 \mathrm{~K}$ and minimum at $\Delta \omega \approx 1.1 \mathrm{~K}$ of the plasmon spectrum (cf. Fig. 3) and show the single-peak behavior of $S(\vec{q}, \omega)$. The dashed-dot-dot and dashed-dot lines illustrate the splitting of the structure factor peaks for $\Delta \omega \approx 0.25$ and $1.5 \mathrm{~K}$. The inset shows the asymmetric double-peak structure of the structure factor for $\Delta \omega \approx 0.7 \mathrm{~K}$.

tween two subregions of the EHC is crossed at $\phi_{q} \simeq \pi / 2$. Entering the new region, the plasmon becomes overdamped, concurrent with the sharp rise of $\operatorname{Im} \varepsilon(\vec{q}, \omega)$, which we plot in the same figure on the right axis. Figure 3 shows that there are two ranges of directions $\pi / 2 \lesssim \phi_{q} \lesssim 5 \pi / 8$ and $7 \pi / 8$ $\lesssim \phi_{q} \lesssim \pi$ in which the plasmon cannot propagate due to excessive Landau damping. On the other hand, the plasmon is well defined around the angles $\phi_{\mathbf{q}}=\pi / 4$ and $3 \pi / 4$, where the imaginary part of $\varepsilon(\vec{q}, \omega)$ is small. These are the principal directions of the underlying structural $C_{2 v}$ symmetry. Finally, in the intermediate II region in Fig. 2, the intra- and interchirality subbands either overlap or not so that the plasmon is being either damped or not depending on its propagation direction.

In Fig. 4 we plot the dynamical structure factor $S(\vec{q}, \omega)$ $=-\operatorname{Im}[1 / \varepsilon(\vec{q}, \omega)]$ as a function of $\phi_{\mathbf{q}}$ for $\omega$ corresponding to the local maximum and minimum of the plasmon energy dispersion. In both cases $S(\vec{q}, \omega)$ shows a dominant peak as a function of $\phi_{q}$. As expected, the peak occurs at $\phi_{\mathbf{q}}=\pi / 4$ for $\omega=\omega_{\min }(q)$ (dashed line) and at $\phi_{q}=3 \pi / 4$ for $\omega=\omega_{\max }(q)$ (solid line). These peaks represent lightly Landau damped plasmons (the plasmon at $\phi_{q}=3 \pi / 4$ is less damped than the one at $\phi_{q}=\pi / 4$ and therefore produces a stronger peak). The preferential role of these two directions comes from the $C_{2 v}$ symmetry of the problem, clearly seen from the plot of the Fermi surface in Fig. 1. Notice that for a given $\omega$ there are two additional angles at which $\operatorname{Re} \varepsilon(\vec{q}, \omega)$ shows zeros. The structure factor, however, does not exhibit peaks at these angles since the large density of electron-hole pairs reflected in the large value of $\operatorname{Im} \varepsilon(\vec{q}, \omega)$ overdamps the plasmons in these directions. These "overdamped plasmons" are represented by the triangle symbols in Fig. 3 .

In the range $\omega_{\max }(q)<\omega<\omega_{\min }(q)$ between the extrema of the plasmon spectrum, the height and the width of the peaks of $S(\vec{q}, \omega)$ vs $\phi_{q}$ show a smooth evolution: with increasing $\omega$ one peak diminishes, the other grows, and vice versa. Thus, in this intermediate region the structure factor has two peaks located at $\phi_{\mathbf{q}}=\pi / 4$ and $3 \pi / 4$, which constitute an asymmetric doublet, shown in the inset of Fig. 4. In the energy regions above the minimum or below the maximum of the plasmon spectrum at given $q$ [i.e., for $\omega$ $>\omega_{\min }(q)$ or $\left.\omega<\omega_{\max }(q)\right]$, $\operatorname{Re} \varepsilon(\vec{q}, \omega)$ vs $\phi_{\mathbf{q}}$ shows two zeros around $\pi / 4$ or $3 \pi / 4$ so that each peak of $S(\vec{q}, \omega)$ splits into two peaks located symmetrically above and below the angle $\phi_{\mathbf{q}}=\pi / 4$ (the dash-dot line) or $3 \pi / 4$ (the dash-dot-dot line).

In the case of $\alpha= \pm \beta$ [see Fig. 1(b)] the linear spin-orbit couplings do not affect the plasmon spectrum: the plasmon damping vanishes and the structure factor is a delta function for all momentum orientations. For this special case there is a global spin-quantization axis-one of the principal $C_{2 v}$ axes-so that the electron gas is split into two uncoupled spin components whose circular Fermi contours are shifted from the origin in opposite directions. Each component gives an isotropic collective response, as guaranteed by Galilean invariance. Cubic spin-orbit terms which spoil this effect are typically much weaker in quantum wells.

In conclusion, we have shown that plasmon dynamics (spectrum and damping) is strongly anisotropic in realistic zinc-blende quantum wells due to the interplay of two different forms of spin-orbit interaction. Experimental observation of this anisotropy would be of fundamental interest and could open the way to new techniques for controlled directional plasmon filtering, potentially useful for both spintronic and plasmonic devices.

This work is supported by the Volkswagen Foundation, SFB under Grant No. 689, NSF under Grant No. DMR0705460, and ANSEF under Grant No. PS-1576. We thank T. Reinecke, C. Schuller, T. Korn, and S. Abedinpour for useful discussions.

\footnotetext{
*samvel.badalyan@physik.uni-regensburg.de

${ }^{1}$ I. Žutić, J. Fabian, and S. Das Sarma, Rev. Mod. Phys. 76, 323 (2004).

${ }^{2}$ J. Fabian, A. Matos-Abiague, C. Ertler, P. Stano, and I. Zutic, Acta Phys. Slov. 57, 565 (2007).

${ }^{3}$ Yu. Bychkov and E. I. Rashba, JETP Lett. 39, 78 (1984).

${ }^{4}$ G. Dresselhaus, Phys. Rev. 100, 580 (1955).
}

${ }^{5}$ M. I. D'yakonov and V. Yu. Kachorovskii, Sov. Phys. Semicond. 20, 110 (1986).

${ }^{6}$ Y. K. Kato, R. C. Myers, A. C. Gossard, and D. D. Awschalom, Science 306, 1910 (2004).

${ }^{7}$ J. Wunderlich, B. Kaestner, J. Sinova, and T. Jungwirth, Phys. Rev. Lett. 94, 047204 (2005).

${ }^{8}$ N. S. Averkiev and L. E. Golub, Phys. Rev. B 60, 15582 (1999). 
${ }^{9}$ P. Stano and J. Fabian, Phys. Rev. Lett. 96, 186602 (2006).

${ }^{10}$ J. Moser, A. Matos-Abiague, D. Schuh, W. Wegscheider, J. Fabian, and D. Weiss, Phys. Rev. Lett. 99, 056601 (2007).

${ }^{11}$ J. L. Cheng, M. W. Wu, and I. C. da Cunha Lima, Phys. Rev. B 75, 205328 (2007).

${ }^{12}$ J. A. Maytorena, C. López-Bastidas, and F. Mireles, Phys. Rev. B 74, 235313 (2006).

${ }^{13}$ B. A. Bernevig, J. Orenstein, and S. C. Zhang, Phys. Rev. Lett. 97, 236601 (2006).

${ }^{14}$ C. P. Weber, J. Orenstein, B. A. Bernevig, S. C. Zhang, J. Stephens, and D. D. Awshalom, Phys. Rev. Lett. 98, 076604 (2007).

${ }^{15}$ M. Trushin and J. Schliemann, Phys. Rev. B 75, 155323 (2007).

${ }^{16}$ C. P. Weber, N. Gedik, J. E. Moore, J. Orenstein, J. Stephens, D. D. Awschalom, Nature (London) 437, 1330 (2005).

${ }^{17}$ S. M. Badalyan, C. S. Kim, and G. Vignale, Phys. Rev. Lett. 100, 016603 (2008).

${ }^{18}$ C. A. Ullrich and M. E. Flatte, Phys. Rev. B 68, 235310 (2003).

${ }^{19}$ M. Pletyukhov and V. Gritsev, Phys. Rev. B 74, 045307 (2006).

${ }^{20}$ W.-K. Tse and S. Das Sarma, Phys. Rev. B 75, 045333 (2007).

${ }^{21}$ M. S. Kushwaha and S. E. Ulloa, Phys. Rev. B 73, 205306
(2006).

${ }^{22}$ D. S. Saraga and D. Loss, Phys. Rev. B 72, 195319 (2005).

${ }^{23}$ X. F. Wang, Phys. Rev. B 72, 085317 (2005).

${ }^{24}$ G. Gumbs, Phys. Rev. B 72, 165351 (2005).

${ }^{25}$ W. Xu, Appl. Phys. Lett. 82, 724 (2003).

${ }^{26}$ L. I. Magarill, A. V. Chaplik, and M. V. Éntin, JETP 92, 153 (2001).

${ }^{27}$ S. A. Maier, Plasmonics-Fundamentals and Applications (Springer, New York, 2007).

${ }^{28}$ S. D. Ganichev, V. V. Bel'kov, L. E. Golub, E. L. Ivchenko, P. Schneider, S. Giglberger, J. Eroms, J. DeBoeck, G. Borghs, W. Wegscheider, D. Weiss, and W. Prettl, Phys. Rev. Lett. 92, 256601 (2004).

${ }^{29}$ S. Giglberger, L. E. Golub, V. V. Bel'kov, S. N. Danilov, D. Schuh, Ch. Gerl, F. Rohlfing, J. Stahl, W. Wegscheider, D. Weiss, W. Prettl, and S. D. Ganichev, Phys. Rev. B 75, 035327 (2007).

${ }^{30}$ G. F. Giuliani and G. Vignale, Quantum Theory of the Electron Liquid (Cambridge University Press, Cambridge, 2005).

${ }^{31}$ F. Stern, Phys. Rev. Lett. 18, 546 (1967). 